\title{
The Solutions and Periods of Some Considered Non-Linear Difference Equation Systems
}

\author{
Kemal Uslu ${ }^{1, *}$, Vural Deniz ${ }^{2}$ \\ ${ }^{1}$ Selcuk University, Science Faculty, Department of Mathematics \\ Konya, Turkey \\ ${ }^{2}$ Selcuk University, Science Faculty, Department of Mathematics \\ Konya, Turkey \\ "Corresponding author's email: kuslu@selcuk.edu.tr
}

\begin{abstract}
We find the periods of some non-linear discrete equation systems in this study. Then we obtain the solutions of these systems releted to initial values.
\end{abstract}

Keywords - The period of discrete system, high order non-linear discrete systems

\section{INTRODUCTION}

Difference equations system arise in many branches of mathematics as well as other sciences. It is a fascinating issue because it is used to solve some problems that appear to be very complicated in applied areas. It has a wide application area especially in biology, physics, social and engineering fields [1-9]. Some are following. Clark and Kulenovic examined the global stability properties and asymptotic behavior of recursive equation systems at [2]. Nasri M and at all, introduced a deterministic model for HIV infection in the presence of combination therapy with differential equations system [4]. In [1,3], Cınar C., Yalçınkaya I. and Iricanin B., Stevic S. have considered some system of nonlinear difference equations of higher order with periodic solutions. Kose H., Uslu K. and Taskara N., examined the solution dynamics of the nonlinear iteration system in [5]. In [5,7,8,9], some nonlinear discrete systems have been investigated and the dynamics of these systems have been investigated.

In this study, we consider following non-linear systems

$$
\begin{aligned}
& p_{n+1}=\frac{1}{s_{n}-r_{n}-q_{n}}+\frac{1}{q_{n-1}-r_{n-1}}, \quad q_{n+1}=\frac{1}{s_{n}-r_{n}-q_{n}}+\frac{r_{n}\left(s_{n-1}-r_{n-1}-q_{n-1}\right)}{r_{n-1}}, \\
& r_{n+1}=\frac{1}{s_{n}-r_{n}-q_{n}}, \quad s_{n+1}=\frac{2}{s_{n}-r_{n}-q_{n}}+\frac{r_{n}\left(s_{n-1}-r_{n-1}-q_{n-1}\right)}{r_{n-1}}+\frac{1}{p_{n}-r_{n}},(n \geq 0)
\end{aligned}
$$

with initial values $p_{0}, q_{-1}, q_{0}, r_{-1}, r_{0}, s_{-1}, s_{0} \in \mathbb{R}-\{0\}, p_{0} \neq r_{0}, s_{i} \neq q_{i}+r_{i}, q_{i} \neq r_{i}, i \in\{-1,0\}$,

and

$$
\begin{aligned}
& p_{n+1}=\frac{1}{s_{n-1}-r_{n-1}-q_{n-1}}+\frac{1}{q_{n-2}-r_{n-2}}, \quad q_{n+1}=\frac{1}{s_{n-1}-r_{n-1}-q_{n-1}}+\frac{r_{n}\left(s_{n-2}-r_{n-2}-q_{n-2}\right)}{r_{n-1}}, \\
& r_{n+1}=\frac{1}{s_{n-1}-r_{n-1}-q_{n-1}}, s_{n+1}=\frac{2}{s_{n-1}-r_{n-1}-q_{n-1}}+\frac{r_{n}\left(s_{n-2}-r_{n-2}-q_{n-2}\right)}{r_{n-1}}+\frac{1}{p_{n-1}-r_{n-1}},(n \geq 0)
\end{aligned}
$$

with initial values $p_{-1}, p_{0}, q_{-2}, q_{-1}, q_{0}, r_{-2}, r_{-1}, r_{0}, s_{-2}, s_{-1}, s_{0} \in \mathbb{R}-\{0\}, p_{i} \neq r_{i}, i \in\{-1,0\}, s_{i} \neq q_{i}+r_{i}, q_{i} \neq r_{i}, i \in$ $\{-2,-1,0\}$.

Firstly, we give basic definitions. Let $\mathrm{I}_{1}, \mathrm{I}_{2}, \mathrm{I}_{3}$ and $\mathrm{I}_{4}$ be some intervals of real numbers and let $\mathrm{F}_{1}: \mathrm{I}_{2} \times \mathrm{I}_{3} \times \mathrm{I}_{4} \rightarrow \mathrm{I}_{1}$, $\mathrm{F}_{2}: \mathrm{I}_{2} \times \mathrm{I}_{3} \times \mathrm{I}_{4} \rightarrow \mathrm{I}_{2}, \mathrm{~F}_{3}: \mathrm{I}_{2} \times \mathrm{I}_{3} \times \mathrm{I}_{4} \rightarrow \mathrm{I}_{3}$ and $\mathrm{F}_{4}: \mathrm{I}_{1} \times \mathrm{I}_{2} \times \mathrm{I}_{3} \times \mathrm{I}_{4} \rightarrow \mathrm{I}_{4}$ be four continuously differentiable functions. It is obvious that the system of difference equations (1.3)

$$
\begin{aligned}
& p_{n+1}=F_{1}\left(q_{n}, r_{n}, s_{n}\right), \quad q_{n+1}=F_{2}\left(q_{n}, r_{n}, s_{n}\right) \\
& r_{n+1}=F_{3}\left(q_{n}, r_{n}, s_{n}\right), \quad s_{n+1}=F_{4}\left(p_{n}, q_{n}, r_{n}, s_{n}\right)
\end{aligned}
$$


has a unique solution $\left\{p_{n}, q_{n}, r_{n}, s_{n}\right\}$ for every initial condition $\left(p_{i}, q_{i}, r_{i}, s_{i}\right) \in \mathrm{I}_{1} \times \mathrm{I}_{2} \times \mathrm{I}_{3} \times \mathrm{I}_{4}$.

A solution $\left\{p_{n}, q_{n}, r_{n}, s_{n}\right\}$ of the system of difference equations (1.3) is periodic if there exist a positive integer m such that

$$
p_{n+m}=p_{n}, \quad q_{n+m}=q_{n}, \quad r_{n+m}=r_{n}, \quad s_{n+m}=s_{n}
$$

the smallest such positive integer $m$ is called the prime period of the solution of difference equation system $(1.3)[2,4]$.

\section{THE SOLUTIONS AND PERIODS OF SOME CONSIDERED NON-LINEAR DIFFERENCE EQUATION SYSTEMS}

The following theorems show us the period of solutions of the systems (1.1) and (1.2).

Theorem 2.1. Suppose that $\left\{p_{n}, q_{n}, r_{n}, s_{n}\right\}$ are the solutions of the difference equation system (1.1) with initial values $p_{0}, q_{-1}, q_{0}, r_{-1}, r_{0}, s_{-1}, s_{0} \in \mathbb{R}-\{0\}, p_{0} \neq r_{0}, s_{i} \neq q_{i}+r_{i}, q_{i} \neq r_{i}, i \in\{-1,0\}$. Then all solutions of the system (1.1) are periodic with period 6 .

Proof: From the system (1.1), it is obtained the following equalities

$$
\begin{aligned}
& p_{n+1}=\frac{1}{s_{n}-r_{n}-q_{n}}+\frac{1}{q_{n-1}-r_{n-1}}, \quad q_{n+1}=\frac{1}{s_{n}-r_{n}-q_{n}}+\frac{r_{n}\left(s_{n-1}-r_{n-1}-q_{n-1}\right)}{r_{n-1}} \\
& r_{n+1}=\frac{1}{s_{n}-r_{n}-q_{n}}, \quad s_{n+1}=\frac{2}{s_{n}-r_{n}-q_{n}}+\frac{r_{n}\left(s_{n-1}-r_{n-1}-q_{n-1}\right)}{r_{n-1}}+\frac{1}{p_{n}-r_{n}}, \\
& p_{n+2}=p_{n}-r_{n}+\frac{1}{q_{n}-r_{n}}, \quad q_{n+2}=p_{n}-r_{n}+\frac{1}{r_{n}} \\
& r_{n+2}=p_{n}-r_{n}, \quad s_{n+2}=2\left(p_{n}-r_{n)}+\frac{1}{r_{n}}+q_{n-1}-r_{n-1},\right. \\
& p_{n+3}=\frac{1}{q_{n-1}-r_{n-1}}+\frac{r_{n-1}}{r_{n}\left(s_{n-1}-r_{n-1}-q_{n-1}\right)}, \quad q_{n+3}=\frac{1}{q_{n-1}-r_{n-1}}+s_{n}-r_{n}-q_{n} \\
& r_{n+3}=\frac{1}{q_{n-1}-r_{n-1}}, \quad s_{n+3}=\frac{2}{q_{n-1}-r_{n-1}}+s_{n}-r_{n}-q_{n}+q_{n}-r_{n} \\
& p_{n+4}=\frac{1}{q_{n}-r_{n}}+r_{n}, \quad q_{n+4}=\frac{1}{q_{n}-r_{n}}+\frac{1}{p_{n}-r_{n}} \\
& r_{n+4}=\frac{1}{q_{n}-r_{n}}, \quad s_{n+4}=\frac{2}{q_{n}-r_{n}}+\frac{1}{p_{n}-r_{n}}+\frac{r_{n}\left(s_{n-1}-r_{n-1}-q_{n-1}\right)}{r_{n-1}}, \\
& p_{n+5}=\frac{r_{n-1}}{r_{n}\left(s_{n-1}-r_{n-1}-q_{n-1}\right)}+\frac{1}{\left(s_{n}-r_{n}-q_{n}\right)}, \quad q_{n+5}=\frac{r_{n-1}}{r_{n}\left(s_{n-1}-r_{n-1}-q_{n-1}\right)}+q_{n-1}-r_{n-1}
\end{aligned}
$$




$$
\begin{gathered}
r_{n+5}=\frac{r_{n-1}}{r_{n}\left(s_{n-1}-r_{n-1}-q_{n-1}\right)}, \quad s_{n+5}=\frac{2 r_{n-1}}{r_{n}\left(s_{n-1}-r_{n-1}-q_{n-1}\right)}+q_{n-1}-r_{n-1}+\frac{1}{r_{n}}, \\
p_{n+6}=p_{n}, \quad q_{n+6}=q_{n} \\
r_{n+6}=r_{n}, \quad s_{n+6}=s_{n} .
\end{gathered}
$$

Thus all solutions of the system (1.1) are periodic with 6 period.

Theorem 2.2. Suppose that $\left\{p_{n}, q_{n}, r_{n}, s_{n}\right\}$ are the solutions of the difference equation system (1.2) with initial values $p_{-1}, p_{0}, q_{-2}, q_{-1}, q_{0}, r_{-2}, r_{-1}, r_{0}, s_{-2}, s_{-1}, s_{0} \in \mathbb{R}-\{0\}, p_{i} \neq r_{i}, i \in\{-1,0\}, s_{i} \neq q_{i}+r_{i}, q_{i} \neq r_{i}, i \in\{-2,-1,0\}$. Then all solutions of the system (1.2) are periodic with period 9.

Proof: From the system (1.2), it is obtained the following equalities

$$
\begin{aligned}
& p_{n+1}=\frac{1}{s_{n-1}-r_{n-1}-q_{n-1}}+\frac{1}{q_{n-2}-r_{n-2}}, \quad q_{n+1}=\frac{1}{s_{n-1}-r_{n-1}-q_{n-1}}+\frac{r_{n}\left(s_{n-2}-r_{n-2}-q_{n-2}\right)}{r_{n-1}}, \\
& r_{n+1}=\frac{1}{s_{n-1}-r_{n-1}-q_{n-1}}, \quad s_{n+1}=\frac{2}{s_{n-1}-r_{n-1}-q_{n-1}}+\frac{r_{n}\left(s_{n-2}-r_{n-2}-q_{n-2}\right)}{r_{n-1}}+\frac{1}{p_{n-1}-r_{n-1}}, \\
& p_{n+2}=\frac{1}{s_{n}-r_{n}-q_{n}}+\frac{1}{q_{n-1}-r_{n-1}}, \quad q_{n+2}=\frac{1}{s_{n}-r_{n}-q_{n}}+\frac{1}{r_{n}}, \\
& r_{n+2}=\frac{1}{s_{n}-r_{n}-q_{n}}, \quad s_{n+2}=\frac{2}{s_{n}-r_{n}-q_{n}}+\frac{1}{r_{n}}+\frac{1}{p_{n}-r_{n}}, \\
& p_{n+3}=p_{n-1}-r_{n-1}+\frac{1}{q_{n}-r_{n}}, \quad q_{n+3}=p_{n-1}-r_{n-1}+s_{n-1}-r_{n-1}-q_{n-1}, \\
& r_{n+3}=p_{n-1}-r_{n-1}, \quad s_{n+3}=2\left(p_{n-1}-r_{n-1}\right)+s_{n-1}-r_{n-1}-q_{n-1}+q_{n-2}-r_{n-2}, \\
& p_{n+4}=p_{n}-r_{n}+\frac{r_{n-1}}{r_{n}\left(s_{n-2}-r_{n-2}-q_{n-2}\right)}, \quad q_{n+4}=p_{n}-r_{n}+s_{n}-r_{n}-q_{n}, \\
& r_{n+4}=p_{n}-r_{n}, \quad s_{n+4}=2\left(p_{n}-r_{n}\right)+s_{n}-r_{n}-q_{n}+q_{n-1}-r_{n-1}, \\
& p_{n+5}=\frac{1}{q_{n-2}-r_{n-2}}+r_{n}, \quad q_{n+5}=\frac{1}{q_{n-2}-r_{n-2}}+\frac{1}{p_{n-1}-r_{n-1}}, \\
& r_{n+5}=\frac{1}{q_{n-2}-r_{n-2}}, \quad s_{n+5}=\frac{2}{q_{n-2}-r_{n-2}}+\frac{1}{p_{n-1}-r_{n-1}}+q_{n}-r_{n}, \\
& p_{n+6}=\frac{1}{q_{n-1}-r_{n-1}}+\frac{1}{s_{n-1}-r_{n-1}-q_{n-1}}, \quad q_{n+6}=\frac{1}{q_{n-1}-r_{n-1}}+\frac{1}{p_{n}-r_{n}}, \\
& r_{n+6}=\frac{1}{q_{n-1}-r_{n-1}}, \quad s_{n+6}=\frac{2}{q_{n-1}-r_{n-1}}+\frac{1}{p_{n}-r_{n}}+\frac{r_{n}\left(s_{n-2}-r_{n-2}-q_{n-2}\right)}{r_{n-1}},
\end{aligned}
$$




$$
\begin{gathered}
p_{n+7}=\frac{1}{q_{n}-r_{n}}+\frac{1}{s_{n}-r_{n}-q_{n}}, \quad q_{n+7}=\frac{1}{q_{n}-r_{n}}+q_{n-2}-r_{n-2}, \\
r_{n+7}=\frac{1}{q_{n}-r_{n}}, \quad s_{n+7}=\frac{2}{q_{n}-r_{n}}+q_{n-2}-r_{n-2}+\frac{1}{r_{n}}, \\
p_{n+8}=\frac{r_{n-1}}{r_{n}\left(s_{n-2}-r_{n-2}-q_{n-2}\right)}+p_{n-1}-r_{n-1}, \quad q_{n+8}=\frac{r_{n-1}}{r_{n}\left(s_{n-2}-r_{n-2}-q_{n-2}\right)}+q_{n-1}-r_{n-1}, \\
r_{n+8}=\frac{r_{n-1}\left(s_{n-2}-r_{n-2}-q_{n-2}\right)}{r_{n}} \quad \begin{array}{c}
s_{n+8}=\frac{2 r_{n-1}}{r_{n}\left(s_{n-2}-r_{n-2}-q_{n-2}\right)}+q_{n-1}-r_{n-1}+s_{n-1}-r_{n-1}-q_{n-1}, \\
p_{n+9}=p_{n}, \quad q_{n+9}=q_{n} \\
r_{n+9}=r_{n}, \quad s_{n+9}=s_{n} .
\end{array}
\end{gathered}
$$

Thus all solutions of the system (1.1) are periodic with 9 period.

This study is related to the master's thesis of Vural Deniz.

\section{REFERENCES}

[1] Cinar C., Yalcinkaya I., "On the positive solutions of difference equation system $x_{n+1}=\frac{1}{z_{n}}, y_{n+1}=\frac{1}{x_{n-1} y_{n-1}}, \quad z_{n+1}=\frac{1}{x_{n-1}}$, International Mathematical Journal, vol. 5, 2004.

[2] Clark D., Kulenovic M.R.S., "A coupled system of rational difference equations", Computers and Mathematics with Applications, 43, 849-867, 2002.

[3] Iricanin B., Stevic S., "Some systems of non-linear difference equations of higher order with periodic solutions", Dynamic of Continuous, Discrete and Impulse Sysetems, Series A Mathematical Analysis, vol.13, 499-507, 2006.

[4] Nasri M., Dehghan M. and Douraki M.J., Mathias R., "Study of a system of non-linear difference equations arising in a deterministic model for HIV infection", Applied Mathematics and Computation, 171, 1306-1330, 2005.

[5] Kose H., Uslu K., Taskara N., "On the dynamics of solutions of non-linear recursive system", Communications in Mathematics and Applications, 2(5), 91-98, 2010.

[6] Taskara N., Uslu K., Tollu D. T., "The periodicity and solutions of the rational difference equation with periodic coefficients", Computers \& Mathematics with Applications, 62(4), 1807-1813, 2011.

[7] Tollu D. T., Taskara N., Uslu K., "The periodicity of solutions of the rational difference equation $x_{-}\{n+1\}=\left(\left(p_{-}\{n\} x_{-}\{n-k\}+x_{-}\{n-k-1\}\right) /\left(q_{-}\{n\}+x_{-}\{n-k-1\}\right)\right) ", 14$.th International Congress on Computational and Applied Mathematics, 2009.

[8] Uslu K., Hekimoğlu E. O., "On the non-linear difference equation and its solution", Far East Journal of Mathematical Sciences, 27(3), 473-478, 2007.

[9] Uslu K., Taskara N., Hekimoglu O., "On the periodicity and stability conditions of a non-linear system", The First International Conference on Mathematics and Statistics, American University of Sharjah, UAE, 110 pp., Mart 18-21, 2010. 